\title{
Serious Adverse Reactions to Anti-snake Venom in Children with Snake Envenomation: An Underappreciated Contributor to Snakebite Mortality?
}

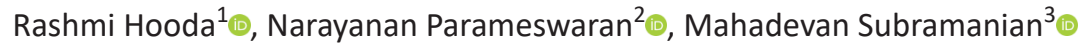

\begin{abstract}
Background: Deaths due to snakebites and serious adverse reactions to anti-snake venom (ASV) are both underreported in India. Serious adverse reactions to ASV are common, contributing significantly to mortality and morbidity. We conducted a study to determine the frequency of occurrence of severe adverse reactions to ASV in children and study the various risk factors and their outcomes.

Patients and methods: We carried out a retrospective record review of all children of snake envenomation admitted in our tertiary care teaching hospital, from January 2013 to December 2016. Children aged 0 to 12 years admitted for snake envenomation and who received ASV as part of their treatment were included. Details about their management, including ASV usage and any adverse effects noted, were collected on a standard data collection form.

Results: Sixty-eight children were enrolled. Hemotoxic (52.9\%) envenomation was more common than neurotoxic (35.2\%). Severe adverse reactions were present in $42.6 \%$, hypotension in $38.2 \%$, and bronchospasm in $4.4 \%$ of the children. The overall mortality rate was $16.1 \%$, anaphylaxis to ASV contributing to $36.3 \%$ of them. Mortality was significantly higher in cases with severe adverse reactions $(p=0.005)$. ASV reactions were also significantly different with different manufacturers.

Conclusions: There is a high frequency of occurrence of severe adverse reactions to ASV resulting in significant morbidity and mortality.

Keywords: Adverse reactions, Anti-snake venom, Horse serum, Snake envenomation.

Indian Journal of Critical Care Medicine (2021): 10.5005/jp-journals-10071-23836
\end{abstract}

\section{INTRODUCTION}

Snakebite is a major neglected health hazard in India, affecting its vast rural population. According to the National Health Profile 2015 , a total of 1,34,980 cases of snakebite with 1,180 deaths were reported across all states and union territories of India from January to December 2013. ${ }^{1}$ These statistics may not reveal the actual burden as snakebites from remote rural areas are largely underreported. The World Health Organization direct estimates credit India with the highest number of deaths due to snakebites in the world, a staggering 35,000 to 50,000 people dying per year, much higher than the reported deaths due to epidemics that capture the attention of the medical fraternity like dengue, influenza, etc. ${ }^{2,3}$

Anti-snake venom (ASV) is the mainstay of the management of snakebite. The ASV used in India is a polyvalent antivenom that acts against "the big four" species, i.e., common krait (Bungarus ceruleus), cobra (Naja naja), Russell's viper (Daboia russelii), and saw-scaled viper (Echis carinatus). ${ }^{4}$ Each milliliter of ASV used in India neutralizes $0.6 \mathrm{mg}$ of dried Indian cobra, $0.6 \mathrm{mg}$ of dried saw-scaled viper, $0.45 \mathrm{mg}$ of dried Russell's viper, and $0.45 \mathrm{mg}$ of dried common krait venom. ASV, being derived from equine serum, can result in adverse immunologic reactions. The frequency of occurrence of adverse reactions has been reported to be variable, ranging from 12 to $60 \% .{ }^{4-7}$ The frequency of occurrence of reactions seems to be higher in India and Sri Lanka as compared to the rest of the world. ${ }^{4}$ The contribution of these reactions to the mortality and morbidity of snake envenomation is not well documented. Hence, we attempted to determine the frequency of occurrence of severe adverse reactions to ASV in children and study the various risk factors and their outcomes.
${ }^{1}$ Department of Pediatrics, JIPMER, Puducherry, India

${ }^{2}$ Department of Pediatrics, Pt. BDS PGIMS, Rohtak, Haryana, India

${ }^{3}$ Department of Pediatrics Sri Venkateshwaraa Medical College Hospital and Research Centre, Puducherry, India

Corresponding Author: Narayanan Parameswaran, Department of Pediatrics, Pt. BDS PGIMS, Rohtak, Haryana, India, Phone: +91 9443458850, e-mail: drnarayananp@gmail.com

How to cite this article: Hooda R, Parameswaran N, Subramanian M. Serious Adverse Reactions to Anti-snake Venom in Children with Snake Envenomation: An Underappreciated Contributor to Snakebite Mortality? Indian J Crit Care Med 2021;25(6):720-723.

Source of support: Nil

Conflict of interest: None

\section{Subjects and Methods}

We carried out a retrospective record review of all children admitted with snake envenomation at our tertiary care teaching hospital, from January 2013 to December 2016. Children aged 0 to 12 years admitted for snake envenomation and who received ASV as part of their treatment were included. Those who received ASV elsewhere and did not require further doses of ASV in our hospital were excluded. The study was approved by the Institutional Ethics Committee.

From the case records, information about the type of snakebite, the dosage of ASV given, adverse reactions to ASV, the demographic profile of the children, duration between snakebite

(-) Jaypee Brothers Medical Publishers. 2021 Open Access This article is distributed under the terms of the Creative Commons Attribution 4.0 International License (https://creativecommons.org/licenses/by-nc/4.0/), which permits unrestricted use, distribution, and non-commercial reproduction in any medium, provided you give appropriate credit to the original author(s) and the source, provide a link to the Creative Commons license, and indicate if changes were made. The Creative Commons Public Domain Dedication waiver (http://creativecommons.org/publicdomain/zero/1.0/) applies to the data made available in this article, unless otherwise stated. 
and ASV administration, and details about treatment received were collected on a standard data collection form. For the purpose of data collection and analysis, adverse reactions were classified into mild and severe reactions. A severe reaction was defined as any life-threatening reaction to ASV requiring administration of intravenous fluid boluses and/or epinephrine and/or steroids. This included hypotension, bronchospasm, and angioedema. Non-life-threatening reactions, such as chills, itching, skin rashes, etc., were considered mild reactions.

The ASV used in our institute was procured from Vins Bioproducts Ltd. Hyderabad, from 2012 to 2015; and Premium Serums and Vaccines Pvt. Ltd., Pune, Maharashtra, from 2015 to 2017. During administration, one vial of ASV was reconstituted to $10 \mathrm{~mL}$ with sterile water for injection. The usual initial dose was 10 vials in $500 \mathrm{~mL}$ of normal saline, given intravenously without any prior sensitivity testing at a constant rate over a period of 1 hour. Premedication with subcutaneous adrenaline and chlorpheniramine was administered prior to ASV administration. ASV was given under constant monitoring of vital signs, either in an emergency department or in PICU. Children who developed minor reactions were treated immediately with intravenous hydrocortisone and chlorpheniramine. And those who developed major reactions received adrenaline, intravenous fluids if hypotensive, and hydrocortisone. ASV was discontinued in all children who developed reactions. Once the manifestations of the reaction have subsided, ASV was administered at a slower rate under the cover of hydrocortisone and occasionally adrenaline. Children who had cellulitis received appropriate antibiotics and anti-inflammatory drugs after the completion of ASV infusion.

The frequency and type of reactions observed were summarized as proportions. Children who developed adverse reactions were compared with those who did not, and possible risk factors for adverse reactions were identified. For continuous data, student's $t$-test (normally distributed data) and Mann-Whitney $U$ test (if not normally distributed) were used to test the significance of any differences observed. In case of proportions, chi-square test was used. A two-sided $p$ value of $<0.05$ was considered statistically significant. Statistical analysis was carried out using Epi Info 3.5.4.

\section{Results}

During the study period of 3 years, 83 children were admitted with snake envenomation. Out of all admitted children, 15 had received ASV from the referring hospital and did not receive further ASV in our hospital and were thus excluded. The demographic profile of the remaining 68 children who constituted the study population is depicted in Table 1, and the clinical features are shown in Figure 1.

The median time to reach the hospital of first contact was 60 minutes [interquartile range (IQR), 45-120]. Out of the 83 children

Table 1: Demographic profile and baseline characteristics of study children $(N=68)$

\begin{tabular}{ll}
\hline Variable & $n(\%)$ \\
\hline Age (years) : $0-5$ & $21(30.8)$ \\
$6-12$ & $47(69)$ \\
Gender: Boys & $36(53)$ \\
Type of bite (clinical features) & \\
• Hemotoxic & $36(52.9)$ \\
- Neurotoxic & $24(35.2)$ \\
• Both hemotoxic and neurotoxic & $4(5.8)$ \\
• Only local manifestations & $4(5.8)$ \\
\hline
\end{tabular}
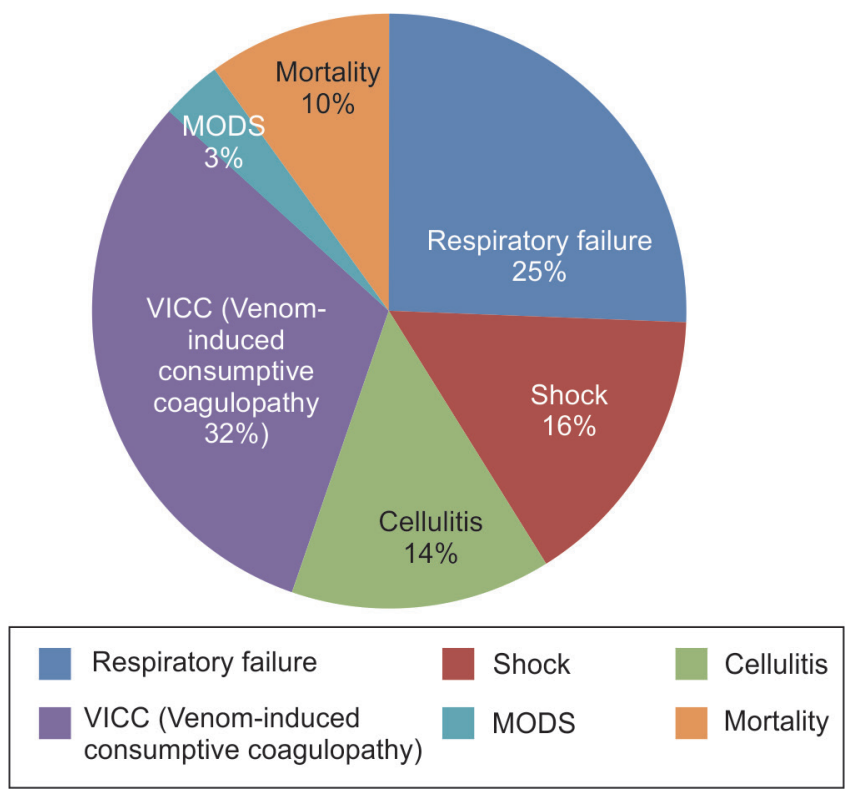

Fig. 1: Clinical characteristics of the study children

admitted with snakebite, 23 (33.8\%) received ASV before reaching our hospital. Out of the 23 who received ASV outside, eight received further doses in our hospital and 15 did not receive any more doses of ASV. The median dose of ASV used outside our hospital was eight vials (IQR, 5-10). The median time to reach our hospital post-snakebite was 225 minutes (IQR, 150-435). The total dose of ASV received by the study children was $15.8 \pm 6.7$ (mean \pm SD). Out of the 23 children who were initially treated outside with ASV, the anticipation of respiratory failure necessitated referral in seven children, eight were referred due to adverse reactions, like rash, fever, and vomiting, and the cause of referral was not mentioned for the remaining eight children.

Severe adverse reactions were found in 29 (42.6\%) children, of whom 26 had hypotension and three had bronchospasm. Mild reactions were present in 25 children, while chills and rigors being the most common were found in $55.5 \%$ of study children. Itching and urticaria were present in $41 \%$ of children, while nausea, vomiting, and abdominal pain were reported in two patients (Table 2). None of the children had any documented exposure to horse serum in the past.

The comparison between the patients with and without severe adverse reactions is shown in Table 3. We also observed that the manufacturer supplying the ASV to the hospital was different before and after the year 2016. During the period before

Table 2: Adverse reactions after ASV administration in the study children $(N=68)$

\begin{tabular}{lc}
\hline Major adverse reactions $(N=29)$ & $n(\%)$ \\
\hline • Hypotension & $26(38.2)$ \\
• Bronchospasm & $3(4.4)$ \\
\hline Minor adverse reactions $(N=25)^{*}$ & $n(\%)$ \\
\hline • Chills & $17(25)$ \\
• Rigor & $14(20.5)$ \\
• Fever & $9(13.2)$ \\
• Rash & $6(8.8)$ \\
• Itching & $2(2.9)$ \\
• Nausea/vomiting/abdominal pain & $2(2.9)$ \\
\hline *Some children had more than one minor adverse reaction. &
\end{tabular}


Table 3: Comparison of clinical characteristics of children with and without severe ASV reactions

\begin{tabular}{llll}
\hline & \multicolumn{2}{l}{ Severe ASV reaction } & \\
\cline { 2 - 4 } \cline { 2 - 3 } & $\begin{array}{l}\text { Yes } \\
(n=29)\end{array}$ & $\begin{array}{l}\text { No } \\
(n=39)\end{array}$ & $p$ value \\
\hline Hemotoxic $(n=36), n(\%)$ & $16(44.4 \%)$ & $20(55.5 \%)$ & 0.75 \\
Non-hemotoxic $(n=32), n(\%)$ & $13(40.6 \%)$ & $19(59.3 \%)$ & \\
Adrenaline premedication $(n=62), n(\%)$ & $27(43.5 \%)$ & $35(56.4 \%)$ & 0.33 \\
Subjects who received ASV outside $(n=23), n(\%)$ & $10(43.4 \%)$ & $13(56.5 \%)$ & 0.92 \\
No. of vials of ASV received outside $(n=23)$, median $(\mathrm{IQR})$ & $8(3.3)$ & $8(3.5)$ & 0.9 \\
Average no. of total vials used, median (IQR) & $12(10)$ & $11(10)$ & 0.75 \\
Mortality $(n=11), n(\%)$ & $9(81.8 \%)$ & $2(18.2 \%)$ & 0.006 \\
\hline
\end{tabular}

2016, 50\% ( $n=25$ ) of children had severe reactions as compared to $22.2 \%(n=4)$ observed during the year 2016. This difference was statistically significant $(p=0.04)$.

Of the 11 children who expired, the cause of death was attributed directly to severe anaphylaxis in four patients. Out of the remaining seven children, one child died due to sudden hypotension during hemodialysis. Two children were shifted to our hospital in a post-cardiac arrest state and expired as a result of refractory cardiogenic shock. Four children died due to direct complications of snake envenomation; two died of uncontrolled disseminated intravascular coagulopathy and two children of anuric acute kidney injury and pulmonary edema.

\section{Discussion}

Serious reactions to ASV were very common (42.6\%) among our study population and contributed to $36.3 \%$ of the mortality observed. Among the study population, most children (69\%) belonged to the age-group of 5 to 12 years, and boys and girls were equally affected. Though hemotoxic manifestations were more common than neurotoxic features in our study children, we observed that the proportion of children with neurotoxic envenomation was higher in our study as compared to previous studies. ${ }^{8,9}$ Geographical and seasonal differences could partly explain these differences in various studies. It could also be because of referral bias; patients with neurotoxic manifestations are more likely to be referred early due to apprehension of respiratory failure and the requirement of mechanical ventilation. This is also corroborated by the higher occurrence of respiratory failure observed in our study.

In our study, the frequency of occurrence of all adverse reactions was found to be $57.3 \%$, of which severe reactions constituted $74.3 \%$ of all adverse reactions. This is higher than most studies in the literature.,10 This could be partly because of the retrospective nature of our study and probable sketchy documentation of milder reactions in previous studies. Though high overall rates of adverse reactions have been observed by other researchers as well, most were minor reactions and severe life-threatening reactions reported are lesser than what was observed in our study. ${ }^{11,12}$ It is not easy to distinguish the clinical manifestations of snake envenomation from that of a serious ASV reaction in a child with severe systemic envenomation. Thus, many serious reactions may be mistaken for the clinical worsening of the snake envenomation per se, resulting in underreporting. Our results match with previous studies conducted in the same center by Sankar et al. who reported ASV-induced reactions in $40 \%$ of their children, with a mortality rate of $13 \%$, and also by Jayakrishnan et al. who reported ASV-induced reactions in $45.5 \%$ of study children, with a mortality rate of $10.3 \% .^{13,14}$

The overall mortality rate observed in our study (16\%) and deaths attributed to ASV anaphylaxis (36\%) were found to be higher as compared to the previous ones. ${ }^{11,12}$ In our study population, 26 (38.2\%) children had cardiovascular compromise necessitating the use of vasopressors or fluid boluses either at presentation or during the hospital stay. Out of these children, 18 (26.4\%) had a shock at presentation and the rest $(n=8)$ developed shock subsequently, attributed to ASV-induced anaphylaxis. Thus, children with ASV reaction contributed to $30.7 \%$ of all children with shock. Attributing the mortality due to ASV anaphylaxis to that of snakebite itself could have resulted in the higher mortality in our study.

We tried to ascertain the factors associated with the occurrence of severe anaphylactic reactions in our children. The type of envenomation, whether hemotoxic or neurotoxic, was not observed to have any association with serious reactions. This is unlike the findings of Deshpande et al. who found a significantly higher frequency of adverse reactions in patients with hemotoxic features than neurotoxic. ${ }^{5}$ We also did not observe any difference in the frequency of occurrence of reactions with relation to a particular age-group, gender, or administration of ASV prior to referral to our hospital. Contrary to previous studies, the use of adrenaline premedication was not associated with reduced occurrence of severe adverse reactions in our study. ${ }^{6,15}$ This necessitates the need for further good-quality, randomized controlled trials in this area. No cases of late serum sickness were observed in our study.

We also observed a change in the frequency of occurrence of severe reactions with a change in the manufacturer. Though it is only an observed association and may not be causal, it needs to be studied in detail. The higher occurrence of adverse reactions to ASV in our study also raises serious questions about the manufacturing techniques and quality control of the ASV. Morais had pointed out that sera that are incorrectly purified, or with excessive protein load, can contribute to the development of anaphylactic reactions, especially in susceptible individuals. ${ }^{16}$ This may partly explain the difference in the ASV reactions observed with different manufacturers. The amount of ASV being administered can also be decreased if monovalent ASV is available. This can potentially decrease the quantum of exposure to horse serum. Currently, we are administering antivenom against all four snakes to every patient with snake envenomation in India, substantially increasing the amount of horse serum being used for each patient. In this context, it is worthwhile to note that the use of monovalent ASV in Australia 
has led to a significantly reduced incidence of severe anaphylactic reactions. ${ }^{17}$ It will be difficult to gain widespread acceptance of monovalent ASV in India, in the absence of bedside diagnostic aids to identify the offending snake species.

Our study has certain limitations inherent to any retrospective study. Many mild reactions could have been missed due to the lack of proper documentation. The observed associations in our study are only suggestive and need confirmation in future studies. Despite these limitations, we believe that we could capture most of the severe reactions in our study population as these reactions required additional interventions, which were well documented in the case records. Another limitation was the relatively small sample size. A larger sample of children might have helped us in identifying more significant predictors of severe reactions.

\section{Conclusion}

Hence to conclude, adverse reactions to ASV were very common in our study population, occurring in more than half of the children, three-fourths of which were severe and life-threatening. Severe reactions to ASV contributed to more than one-third of the mortality in our study children. There could be an association between the occurrence of ASV and the manufacturing process that needs to be studied further.

\section{Key Messages}

- Serious adverse reactions to ASV are quite common and contribute to the mortality of snakebites.

- There could be an association between the occurrence of ASV reactions and the manufacturing process that needs to be studied.

\section{ORCID}

Narayanan Parameswaran (1) https://orcid.org/0000-0002-8297-5789

Rashmi Hooda (1) https://orcid.org/0000-0001-8352-6216

Mahadevan Subramanian @ https://orcid.org/0000-0002-3052-6949

\section{References}

1. CBHI. National Health Profile. Central Bureau of Health Inteilligence - Ministry of Health and Family Welfare; 2015. p. 26-38.

2. Chippaux, JP. (1998). Snake-bites : appraisal of the global situation / JP. Chippaux. Bull World Health Org 1998 ; 76(5) : 515-524 https:// apps.who.int/iris/handle/10665/56029

3. Kasturiratne A, Wickremasinghe AR, De Silva N, Gunawardena NK, Pathmeswaran A, Premaratna R, et al. The global burden of snakebite: a literature analysis and modelling based on regional estimates of envenoming and deaths. PLoS Med 2008;5(11):e218. DOI: 10.1371/ journal.pmed.0050218.

4. Warrell DA. WHO/SEARO Guidelines for the clinical management of snake bites in the Southeast Asian region. Southeast Asian J Trop Med Public Health. 1999;30 Suppl 1:1-85. PMID: 10983553.

5. Deshpande RP, Motghare VM, Padwal SL, Pore RR, Bhamare CG, Deshmukh VS, et al. Adverse drug reaction profile of anti-snake venom in a rural tertiary care teaching hospital. J Young Pharm 2013;5(2):41-45. DOI: 10.1016/j.jyp.2013.02.003.

6. Accessed through: https://www.rjpbcs.com/pdf/2013_4(4)/[12].pdf

7. Adhisivam B, Mahadevan S. Snakebite envenomation in India: a rural medical emergency. Indian Pediatr. 2006 Jun;43(6):553-554. PMID: 16820669.

8. Patil V, Patil H, Patil A, Agrawal V. Clinical profile and outcome of envenomous snake-bite at tertiary care centre in western Maharashtra. Int J Med Public Heal 2011;1(4):28-38. DOI: 10.5530/ ijmedph.4.2011.7.

9. Kumaravel KS, Ganesh J. A study on the clinical profile of children with snake envenomation in a tertiary referral centre at Dharmapuri, Tamil Nadu, India. Int J Res Med Sci 2016;4(6):2142-2145. DOI: 10.18203/2320-6012.ijrms20161775.

10. Pore SM, Ramanand SJ, Patil PT, Gore AD, Pawar MP, Gaidhankar SL, et al. A retrospective study of use of polyvalent anti-snake venom and risk factors for mortality from snake bite in a tertiary care setting. Indian J Pharmacol 2015;47(3):270-274. DOI: 10.4103\%2F02537613.157117.

11. Amin M, Mamun S, Rashid R, Rahman M, Ghose A, Sharmin S, et al. Anti-snake venom: use and adverse reaction in a snake bite study clinic in Bangladesh. J Venom Anim Toxins Incl Trop Dis 2008;14(4):660-672. DOI: 10.1590/S1678-91992008000400009.

12. Poovazhagi V, Ravikumar SA, Jagadeeshwari A, Arulganesh P, Raj PS, Anupama S. Anti-snake venom induced reactions among children with snake envenomation. Int J Contemp Pediatr 2017;4(2):629-634. DOI: 10.18203/2349-3291.ijcp20170722.

13. Sankar J, Nabeel R, Sankar MJ, Priyambada L, Mahadevan S. Factors affecting outcome in children with snake envenomation: a prospective observational study. Arch Dis Child 2013;98(8):596-601. DOI: 10.1136/archdischild-2012-303025.

14. Jayakrishnan MP, Geeta MG, Krishnakumar P, Rajesh TV, George B. Snake bite mortality in children: beyond bite to needle time. Arch Dis Child 2017;102(5):445-449. DOI: 10.1136/archdischild2016-311142.

15. Raghuraman MS, Sivaraman B. Comparative study of anaphylaxis incidence in patients receiving anti- snake venom with or without prophylactic adrenaline : a prospective, randomized, blinded study. Int J Basic Clin Pharmacol 2016;5(4):1436-1440. DOI: 10.18203/23192003.ijbcp20162449.

16. Morais $\mathrm{V}$, Massaldi $\mathrm{H}$. Snake antivenoms: adverse reactions and production technology. J Venom Anim Toxins Incl Trop Dis 2009;15(1):2-18. DOI: 10.1590/S1678-91992009000100002.

17. Sutherland SK. Antivenom use in Australia. Premedication, adverse reactions and the use of venom detection kits. Med J Aust. $1992 \mathrm{Dec}$ 7-21;157(11-12):734-739. PMID: 1360618. 